\title{
Research of Port Machinery's Maintenance Scheduling Based on Service Age with Multiple Maintenance Modes Concern
}

\author{
Xiaowei Shi, Huipeng Song, Danping Lin* \\ Logistics Engineering College, Shanghai Maritime University, Shanghai, China \\ Email address: \\ xwshi@shmtu.edu.cn (Xiaowei Shi), happy_ismu@163.com (Huipeng Song),dplin@shmtu.edu.cn (Danping Lin) \\ ${ }^{*}$ Corresponding author
}

\section{To cite this article:}

Xiaowei Shi, Huipeng Song, Danping Lin. Research of Port Machinery's Maintenance Scheduling Based on Service Age with Multiple Maintenance Modes Concern. Science Innovation. Vol. 5, No. 1, 2017, pp. 63-69. doi: 10.11648/j.si.20170501.21

Received: December 30, 2016; Accepted: March 6, 2017; Published: March 15, 2017

\begin{abstract}
Equipment management is important for enterprise management, and maintenance management for the equipment is the core of equipment management. In the equipment-intensive port enterprises, the efficiency of equipment maintenance and management of port machinery directly affects the fluency of port operations, which becomes an important indicator of port competition. Among the current maintenance management theories, the reliability-centered theory is attracting more and more attention. In practical decision, in order to reasonably arrange the maintenance and lessen the number of equipment's downtime, we plan to use different maintenance modes for equipment maintenance. In this paper, we propose multi-mode maintenance for the port equipment based on the service-age analysis. According to the workload of the port machinary, the maintenance mode is selected rationally to minimize the total maintenance cost as well as satisfy the operation capacity of the port. The total maintenance cost includes the preventive maintenance cost, repair cost and replacement cost. In this paper, the model is solved by enumeration method, and the optimal solution is obtained. In addition, the model is tested by several numerical examples.
\end{abstract}

Keywords: Service-Age Analysis, Multiple Maintenance Modes, Failure Rate

\section{基于役龄分析的考虑多种维修模式的港机维修调度研究}

施笑畏, 宋会鹏, 林丹萍*

物流工程学院, 上海海事大学, 上海, 中国

邮箱

xwshi@shmtu. edu.cn（施笑畏），happy_ismu@163.com（宋会鹏），dplin@shmtu. edu. cn（林丹萍）

摘要：设备管理是企业管理的重点, 设备的维修管理又是设备管理的核心。在设备密集的港口企业, 港机设备维修管 理水平的高低直接影响着港口作业的流畅程度, 成为港口竞争的一项重要指标。在当今众多的维修管理理论中, 以可 靠性为中心的理论越来越受到人们的关注。实践决策中, 为了合理的安排维修, 应尽量减少设备停机, 决策时一般需 要采取不同的维修方式对设备进行维修。本文基于役龄分析建立了关于港机多方式维修的优化模型, 根据港机的工作 任务, 在保证设备的生产能力的情况下, 制定合理地生产和维修计划, 使得总维修成本最小。本文的目标函数是最小 化总维修成本, 总维修成本其中包括预防性维修成本、小修成本和更换成本。文中通过采用枚举法对模型进行求解, 求出模型最优解。然后通过一定的算例对模型进行测试。

关键词: 役龄分析, 多种维修方式, 故障率 


\section{1. 引言}

港口企业竞争的核心是作业效率的竞争。机械设备是 港口生存、维持生产的主要力量, 是港口发展的根基, 是 企业取得竞争优势的关键。古语云: 工欲善其事必先利其 器。只有提高机械设备的管理与维修水平, 发挥设备的最 大效能，才能保证港口企业的经营以及生产的高效运行。 如何有效地对机械设备进行科学化管理, 尤其是通过维修 管理和维修策略的制定，保证港口的作业效率，获得最佳 的经济效益, 这已成为港口企业的一项重要管理工作。因 此, 对港口机械维修策略的研究重点关注的方面是以可靠 性为中心的维修, 即为 Reliability Centered Maintenance, 简称RCM。在实际的设备操作过程中, 机械 设备会突然发生故障或因运行时间过长导致设备的可靠 度降低, 从而影响整个工作安排, 导致企业经济效益受损。 为了减少这种情况的发生, 企业一般会提高机械设备检修 的频率, 但这样会增加机械设备的维修成本, 并且会对已 有的工作安排造成影响。所以, 在实际生产过程中, 我们 需要对生产计划和维修计划进行合理地安排。

根据维修的时间划分, 维修大致可分为预防性维修 (Preventive maintenance, 简称 PM) 和事后维修 (Corrective maintenance, 简称CM)。事后维修是机器 发生故障后进行的维修, 通过维修使发生故障的机器可以 继续工作。事后维修是一种传统的维修方式, 事后维修增 加了因设备故障造成的成本, 并且事后维修不能保证设备 的可靠度保持在一定水平上, 影响生产的正常进行。预防 性维修是在设备故障前进行预防性的维修, 预防性维修降 低了机械设备的故障概率, 提高了设备的可靠性, 减少了 应付突发情况的成本。现如今越来越多人研究预防性维修, 特别是预防性维修与生产计划相结合。例如, 在2015年, Ba Kader等 [1]研究了基于生产计划的备件库存与预防性 维修联合优化; Lei Xiao等 [2]对生产安排与成组机器的 预防性维修计划进行联合优化。

近些年来, 一些学者不断对维修策略进行研究, 并提 出了不同的维修策略, 主要包括固定周期的维修策略和依 据设备可靠性而制定的维修策略。金玉兰、蒋祖华等 [3] 研究了以可靠性为中心的预防性维修计划和生产计划优 化问题。另外, 根据设备的维修后效果可以将维修活动大 体分为完备维修、小修和非完备维修。完备维修是指设备 经过维修后设备到达全新状态; 小修是指设备通过维修后 设备恢复到故障前状态; 非完备维修是指设备经过维修后, 故障消除, 设备恢复到一个较新的状态, 介于完备维修和 小修之间。

\section{2. 文献综述}

近些年来, 由于制造业越来越趋向机械化, 机械故障 可能会妨碍生产安排, 给企业带来巨大损失, 所以很多研 究是关于生产与预防性维修, 从整体考虑生产计划和预防 性维修计划, 制定新的生产安排。M. Assid等 [4]研究了利 用产品的准备时间进行机会维修的问题，在考虑机会维修 的情况下对生产和预防性维修进行联合优化。喻明让等 [5]
提出了一种基于调整时间的作业车间调度与预防性维修 集成方法, 通过考虑生产计划安排, 制定合理的维修计划。 崔维伟和陆志强 [6] 针对单机系统, 提出一种综合考虑生 产调度和设备维护的联合决策模型, 联合优化生产和维修 部门的目标, 决定工件的加工顺序和机器维修的方式。金 玉兰等 [7]在单设备系统中, 对设备预防性维修和生产调 度进行多目标优化。王世进 [8]研究了一种单机环境下集 成生产和维护的双目标优化调度问题。以上这些维修理论 主要是关于维修计划与生产安排的优化问题, 虽然是从生 产计划和维修计划整体考虑, 但其中没有考虑到设备的役 龄对设备自身的影响, 以及对设备维修和生产的影响。

除了从生产的角度考虑维修问题外, 相关研究还从机 械的寿命或可用度进行分析, 对机械的维修计划进行优化。 总的来说, 相对于生产与维修的优化, 从设备本身考虑进 行设备维修优化的研究较少。石慧等 [9]针对退化分布函 数难以估计的复杂系统, 研究了剩余寿命预测及预防性维 护维修最优决策问题。夏唐斌等 [10]结合故障率调整因子, 进行设备寿命分析, 按有效度最大的原则确定了设备的最 佳预防维修周期。张民悦等 [11] 针对故障小修的周期维修 策略, 以部件的稳态可用度、平均费用率和平均可靠度三 个指标构建了一个预防维修周期的多目标最优化模型。王 远航等 [12]基于多故障模式的复杂机械设备, 利用故障时 间分布对设备的预防性维修决策进行优化。米根锁等 [13] 通过收集设备的历史失效数据, 计算设备的剩余寿命并进 行可靠性分析, 进而对其维修计划进行优化。这些文献是 通过对设备的寿命分析，设定设备可靠性阈值或工作时间 阈值, 当设备工作达到阈值时, 就进行维修。

虽然, 有一部分学者在考虑生产计划和维修计划的同 时, 考虑了设备役龄对设备的影响, 但这类文献较少。崔 维伟等 [6] 以单机系统为研究对象, 考虑机器和工件的堕 化效应, 综合决策工件加工顺序和预防性维护时间, 以工 件流程时间最短化和维修成本最小化为联合优化目标。他 在文中考虑到设备的役龄, 他认为机器的役龄与实际加工 时间有关, 当机器役龄越大, 工件的实际加工时间越长。 在对设备役龄分析时, 本文与其不同, 本文考虑的是役龄 对设备的可靠度函数的影响以及维修对设备寿命分布函 数的影响, 进而对维修计划产生影响。

本文在整体考虑生产和维修计划时, 还要考虑到港机 的役龄对其产生的影响, 采用预防性维修、事后维修的小 修和更换多种维修方式来制定港机的维修计划。在对港机 役龄分析的基础上, 分析港机的寿命分布的变化, 在港机 的运行过程中, 不同的维修方式和维修次数, 会对港机的 可靠度产生不同的影响, 在此基础上研究港机多维修方式 的生产和维修的优化问题, 并建立相应的数学模型。与其 他文献不同的是, 本文考虑到维修方式和维修次数对港机 的可靠度的影响, 在考虑港机的役龄分析的基础上, 对港 机的生产和维修计划进行整体优化。

\section{3. 问题描述}

针对单机系统, 在本文中, 生产任务已知, 有n项生产 任务等待在同一个设备上进行同一项的加工操作, 每项生产 
作业中每个作业单位在设备上的操作时间是相同的, $\mathrm{s}_{\mathrm{i}}$ 为第 $\mathrm{i}$ 项作业开始工作的时间, $\mathrm{p}_{\mathrm{i}}$ 为加工第 $\mathrm{i}$ 项的加工时间, $\mathrm{q}_{\mathrm{i}}$ 为第 i项生产作业的权重, 即工作量, 生产作业的加工时间与其 权重成正比。设备从零时刻开始加工, 此时设备处于全新状 态, 随着加工时间的增长, 设备的可靠性会降低, 设备发生 故障的次数会增加, 所以当设备的可靠性降到一定程度时, 为了减少设备故障对生产的影响, 设备需要根据不同状况进 行维修。另外, 设备在生产过程中, 会发生意外故障, 根据 设备故障后其自身的情况可以选择不同的维修方式, 进而使 设备能够继续进行生产。本文就是在已知生产任务的情况下, 根据设备的可靠性和发生故障情况, 合理安排设备的维修和 生产计划, 以达到设备的总维修成本最小。

文中模型还具有以下假设:

（1）预防性维修不可中断每项作业任务，预防性维 修安排在作业任务与作业任务的间隔处。

(2)对于设备的维修方式的选择存在两个阈值, $\alpha$ 为 维修下阈值, $\mu$ 为上阈值。设备的可靠性为 $R(t)$, 当设备 的可靠性 $\mu<R(t) \leq \alpha$ 时, 设备选择预防性维修, 使设备 的状态比之前要好些, 但不是全新状态; 若设备的可靠度 $0 \leq R(t) \leq \mu$ 时, 设备进行更换, 更换后设备达到全新状 态。同样, 当设备发生故障时, 若设备的可靠性 $R(t) \geq \mu$ 时, 设备选择小修, 使设备达到故障前的状态; 反之, 则 进行更换，设备达到全新状态。

(3) 不同的维修方式, 维修成本不同, 它们之间的 大小关系为: 预防性维修<小修<更换, 其中故障状态下的 更换成本要高于非故障状态下的更换成本。

（4）不同的维修方式造成的维修结果不同，进而影 响后续的维修计划, 可能会改变后边的预防性维修时间或 次数。

\section{4. 模型建立}

\section{1. 港机的役龄分析}

威布尔分布是在可靠性技术中应用最广泛的一种分 布。它可用于处理大部分机械零部件, 特别是受变载荷作 用的零部件的疲劳寿命问题。威布尔分布能够很好地描述 港口机械零件的疲劳寿命和强度, 夏唐斌等 [10]在对基于 RCM的港口机械的维修策略研究中, 港口机械零件的疲劳 寿命和强度就是采用威布尔分布; 宋之杰等 [14]在对港机 维修模型研究中, 港机的故障分布也是服从威布尔分布。 因此, 本文也采用威布尔分布进行研究。其故障率为:

$$
\lambda(t)=\frac{\beta}{\eta}\left(\frac{t}{\eta}\right)^{\beta-1}
$$

( $\beta$ 为形状参数, $\eta$ 为特征寿命参数) 故障率与可靠度之间的关系如下:

$$
R(t)=e^{-\int_{0}^{t} \lambda(t) d t}
$$

（1）港机在自然状态下进行工作，不对其进行预防 性维修活动, 则港口机械零件的疲劳寿命和强度服从上述 的威布尔分布, 由此可推断出其的可靠度为:

$$
R(t)=e^{-\left(\frac{t}{\eta}\right)^{\beta}}
$$

港机从零时刻开始工作时, 港机的可靠性分析符合以 上函数分布, 港机的可靠度与其工作时间的关系为以上函 数关系。

（2）在现实情况下, 为了港机能够正常工作, 需要 对其进行维修, 这时, 港机的寿命分布就会与自然状态下 的不同，港机的故障率会发生改变。

Malik首先提出了役龄递减因子的概念 [15]。他认为 在第 $i$ 次预防维护之后, 设备的故障率模型将变成: $\lambda_{i+1}(t)=\lambda_{i}\left(t+a T_{i}\right) t \in\left(0, T_{i+1}\right), 0<a<1$ 为役龄递减因子, $T_{i}$ 为第 $i$ 次维修周期, $\lambda_{i}$ 为第 $i$ 次维修时的故障率, $\lambda_{i+1}(t)$ 为 第 $i+1$ 次维修周期的故障率。Nakagawa提出了故障率递增 因子的概念: $\lambda_{i+1}(t)=b \lambda_{i}(t) t \in\left(0, T_{i+1}\right), b>1$ 为故障率递增 因子 $[16]$ 。

夏唐斌等 $[10]$ 在以上两人的基础上提出了一个考虑 两者优点的故障率演变模型。本文采用其提出的模型, 具 体如下:

$$
\lambda_{i+1}(t)=b \lambda_{i}\left(t+a T_{i}\right) \quad t \in\left(0, T_{i+1}\right)
$$

本文的维修方式主要包括预防性维修、小修和更换。 不同的维修方式, 其产生的维修结果是不同, 当设备进行 更换操作时, 设备的故障率和可靠度函数均与设备最开始 的时候是一致的。对于预防性维修和小修, 预防性维修后 设备的可靠度比维修前的要高一些, 而小修后设备的可靠 度与故障前是一样的。设备每当进行这两种维修后, 其故 障率就会发生如上所说的变化。

故障率演变下设备可靠度公式: 如果设备进行更换后, 处于全新状态, 可靠度为 1 , 故不需要具体计算。但对于 预防性维修和小修, 设备的可靠度计算需要依据上一次维 修前后的可靠度进行计算。

为了便于计算, 本文采用积分中值定理 [17]对公式进 行简化, 得出

$$
\int_{0}^{T_{x}} \lambda_{i+1}(t) d t=\bar{\lambda}_{i+1} \times T_{x}
$$

$\mathrm{T}_{\mathrm{x}}$ ：当故障率为 $\lambda_{i+1}(t)$, 达到已知可靠度设备所需 要运行的时间;

$\bar{\lambda}_{i+1}$ : 第 $\mathrm{i}+1$ 次维修周期内的平均故障率。

进而通过可靠度与故障率之间的关系计算出 $\mathrm{T}_{\mathrm{x}}$, 其中 $R_{i+1}^{0}$ 表示第 $\mathrm{i}+1$ 次维修周期起始时刻的设备可靠度。

$$
T_{x}=-\ln \left(R_{i+1}^{0}\right) / \bar{\lambda}_{i+1}
$$


综合以上计算可以求出第 $i+1$ 次维修前一刻的设备可 靠度, $T_{i+1}$ 为第 $i+1$ 维修周期, $R_{i}$ 表示第 $i$ 次维修后的设备可 靠度, $R_{i+1}^{\prime}(t)$ 表示进行第 $\mathrm{i}+1$ 次维修时设备可靠度。

$$
R_{i+1}^{\prime}(t)=R_{i} \times e^{-\int_{T_{x}}^{T_{x}+T_{i+1}} \lambda_{i+1}(t) d t}
$$

\section{2. 模型建立}

参数说明:

决策变量:

$$
\begin{gathered}
x_{i}= \begin{cases}2, & \text { 第 } i \text { 个作业前进行预防性维修 } \\
1, & \text { 第 } i \text { 个作业前进行正常工作下的更换 }\end{cases} \\
y_{i j}= \begin{cases}2, & \text { 设备故 障 时的更 换 } \\
1, & \text { 设备 故 障 时 的小修 } \\
0, & \text { 其他 }\end{cases}
\end{gathered}
$$

式中: $i=1,2 \ldots n ; j=1,2, \ldots k$ 。

其他参数:

$X:$ 生产作业序列, $X=\left[x_{1}, x_{2}, x_{3}, \ldots x_{n}\right]$ 。

$x_{i}$ : 第i个作业任务, $i=1,2 \ldots n$ 。

$c_{p}:$ 设备预防性维修成本。

$c_{m}$ : 设备小修维修成本。

$c_{r 1}$ : 设备故障时的更换成本。

$c_{r 2}$ : 设备正常工作时的更换成本。

$k$ :第 $\mathrm{i}$ 个作业任务中发生故障次数。

目标函数: 本文的目标函数 $Z$ 是总维修成本最小, 即:

$$
\begin{aligned}
\min Z & =\sum_{i=1}^{n}\left\{\left[c_{p} \times\left(x_{i}-1\right)+c_{r 2}\left(2-x_{i}\right)\right]\right. \\
& \left.+\sum_{j=1}^{k}\left[c_{m} \times\left(2-y_{i j}\right)+\frac{1}{2} c_{r 1}\left(y_{i j}-1\right)\right] \times y_{i j}\right\}
\end{aligned}
$$

s. t.

$$
\begin{gathered}
x_{i}= \begin{cases}2 & i=1,2 \ldots n ; \\
1 & \end{cases} \\
y_{i j}= \begin{cases}2 & i=1,2 \ldots n, \quad j=1,2, \ldots k ; \\
1 & \end{cases}
\end{gathered}
$$

$$
c_{r 1} \geq c_{r 2} \geq c_{m} \geq c_{p} \geq 0 \text { 。 }
$$

公式 (8) 是计算总维修成本的方程, 它是由正常工 作下更换成本、预防性维修成本、小修成本和故障时更换 成本组成; 公式 (9) 是表示正常工作下两种不同的维修 情况, 1 表示当一个工作任务结束后, 设备可靠度小于上 阈值时, 进行更换, 2 表示当一个工作任务结束后, 设备 可靠度小于维修下阈值时, 进行预防性维修; 公式 (10) 是与故障情况下的不同维修方式有关, 0 表示无故障, 1 表示小修，2表示更换; 公式（11）表示所有的单位维修 成本均大于 0 。

\section{5. 算例}

本文是以港口机械为对象进行维修调度的研究, 根据 文献 [10], 我们假设形状参数 $\beta=2$, 特征寿命参数 $\eta=1000 \mathrm{~h}$, 役龄递减因子 $\mathrm{a}=0.15$, 故障率递增因子 $\mathrm{b}=1.15$ 。生产任务 的工作量服从区间为 $[100,599]$ 的离散随机分布, 单位为小 时, 设备故障时刻服从 $[200,699]$ 的离散随机分布, 单位为 小时, 故障程度 $(0,1)$ 随机产生。生产任务的工时与其工作 量成正比。根据实际生产情况, 本文假设预防性维修成本 1000 元/次, 小修成本为 3000 元/次, 在正常工作下的更换 成本为 6000 元/次, 故障情况下的更换成本为 10000 元/次。 同时, 本文假设 $\mu=0.3, \alpha=0.6$, 本文通过枚举法以得出 在不同维修任务规模下, 最小的维修总成本。

从表 1 可以看出, 随着任务个数的增加, 程序的运行 时间越长, 其原因是由于要对任务进行全排列, 造成了运 行次数成指数形式增加, 从而导致运行时间增加。由于当 任务规模为 11 时, 总运行时间超过 24 小时, 不符合维修现 场实际情况的要求, 所以, 本文的计算规模截止到 10 个任 务个数。同时, 我们可以看出当维修任务个数介于 10 和 11 之间时, $n=11$ 的总工作时间小于 $n=10$ 的总工作时间, 但两 者的维修成本一样, 这主要是由于 $n=11$ 时进行了两次故障 后更换 (即更换2)。由表1可知, 故障后更换的成本很高, 所以造成总工作时间小但成本相对来说较高。同时其最小 成本还受单个任务的工作量的影响, 由于每个工作完成或

\begin{tabular}{|c|c|c|c|c|c|c|c|}
\hline \multirow{2}{*}{ 任务数n } & \multirow{2}{*}{ 总工时 $/ h$} & \multirow{2}{*}{ 最小成本Zmin } & \multicolumn{4}{|c|}{ 最小成本时, 维修情况Mmin/次 } & \multirow{2}{*}{ 总运行时间/s } \\
\hline & & & PM & 小修 & 更换1 & 更换2 & \\
\hline 5 & 1879 & 8000 & 2 & 0 & 0 & 1 & 0.078 \\
\hline 6 & 2279 & 11000 & 2 & 1 & 0 & 1 & 0.765 \\
\hline 7 & 2582 & 12000 & 3 & 1 & 0 & 1 & 5. 626 \\
\hline 8 & 2955 & 13000 & 4 & 1 & 0 & 1 & 50.913 \\
\hline 9 & 3058 & 14000 & 5 & 1 & 0 & 1 & $592.166^{\circ}$ \\
\hline 10 & 4080 & 24000 & 5 & 1 & 1 & 1 & 8595.3 \\
\hline 11 & 3755 & 24000 & 6 & 2 & 0 & 2 & 94550.5 \\
\hline
\end{tabular}
开始的节点都会进行维修判断, 如果任务分配恰好在本次 任务结束后且不用进行维修, 但在下一个任务完成后其可 靠度很低，致使其进行更换，那么就会出现总工作时间虽 然小，但相对其他小规模但总工作时间较多的情景，其维 修成本将相对较高。所以，随着任务个数和任务总时间的 增加, 维修成本可能会增加。

表1 不同维修规模下的维修相关结果。

注：更换 1 表示故障情况下，设备故障严重要进行更换；更换 2 表示在正常工作情况下，设备可靠度太低，进行更换。 
本文是对多种维修方式的维修调度问题进行研究，与 其他文献不同，本文通过对多种维修方式进行优化，进而 降低维修成本。下面本文与单纯考虑预防性维修、小修和 机械基本完全失效后换新的维修方式进行对比分析。实验 中假设预防性维修与小修的单位成本与本文相同, 而设备 基本完全失效的换新成本与故障后的更换成本相同。为了
突出实际安排情况，本文只考虑规模任务个数为 5 到 9 之间 的结果对比。

从表 2 可以看出, 在工作任务总量相同的情况下, 采 用本文的维修方法所花费的维修成本要小于采用不包括 更换B的维修方法的维修成本。

表2 不同维修方式下的总维修成本分析。

\begin{tabular}{|c|c|c|c|c|c|c|}
\hline \multirow{2}{*}{ 任务数n } & \multirow{2}{*}{ 总工作时间/h } & \multirow{2}{*}{ 最小成本Zmin } & \multicolumn{4}{|c|}{ 最小成本时, 维修情况矩阵Mmin/次 } \\
\hline & & & PM & 小修 & 更换A & 更换B \\
\hline 5 & 1907 & 8000 & 2 & 0 & 0 & 1 \\
\hline 5 & 1907 & 12000 & 2 & 0 & 1 & -- \\
\hline 6 & 2343 & 11000 & 2 & 1 & 0 & 1 \\
\hline 6 & 2343 & 15000 & 2 & 1 & 1 & -- \\
\hline 7 & 3341 & 11000 & 2 & 1 & 0 & 1 \\
\hline 7 & 3341 & 16000 & 3 & 1 & 1 & - \\
\hline 8 & 2955 & 12000 & 3 & 1 & 0 & 1 \\
\hline 8 & 2955 & 16000 & 3 & 1 & 1 & -- \\
\hline 9 & 3715 & 18000 & 3 & 1 & 0 & 2 \\
\hline 9 & 3715 & 27000 & 4 & 1 & 2 & - \\
\hline
\end{tabular}

注：更换 $\mathrm{A}$ 表示故障情况下，设备基本完全失效进行的换新；更换 $\mathrm{B}$ 表示在正常工作情况下，设备可靠度太低，进行更换。

在任务个数为 7 , 随机产生的任务为 $\mathrm{f}=[507,552,163,556,416,149,239]$, 依据此种情况对参 数 $\mu$ 和 $\alpha$ 进行分析, 通过改变参数, 来观察两个参数对结 果的影响。由于如果预防性维修阈值过大将造成维修次数 过多, 过小会造成欠维修, 所以假设参数 $\alpha$ 其在 $[0.5,0.9]$ 之间进行改变; 同理, 如果更换阈值过大, 会造成更换次 数增加, 增加维修成本而造成资源浪费; 相反, 更换阙值 过小会使设备长期处在可靠度较小的情况下工作, 影响工 作效率。所以参数 $\mu$ 不能过大, 假定其在区间 $(0,0.4]$ 之间进行改变。

通过表 3 , 我们可以看出表 1 中序号 $[1,5] 、[6,10]$ 、 $[11,15]$ 分别对应 $\mu=0.3 、 0.2 、 0.1$, 并且三者之间都是 $\alpha$ 在区间 $[0.8,0.9]$ 之间成本发生了变化, 均多进行了一次预
防性维修, 其主要原因是：当参数 $\alpha$ 过大时, 在进行最后 一部分任务后, 设备的可靠度恰好落入预防性维修的范围。 另外, 从表 3 可知, 当参数 $\mu=0.4$ 时, 通过改变参数 $\alpha$ 而造成的结果与前三种不同, 其相对于其他参数 $\alpha=0.8$ 而言, 多进行了一次预防性维修, 其主要是因为参数 $\mu$ 过 高, 设备进行更换的几率就大, 更换的成本比预防性维修 成本大很多, 所以如果两个任务完成后分别进行预防性维 修与两个任务一起完成后进行一次更换的情况相比, 前者 的成本较小; 当参数 $\mu$ 过高时, 出现两个任务一起完成后 要进行一次更换的情况几率较高, 于是就采用进行两次预 防性维修的方法, 故出现了表1中序号19的情况。而表1 中序号 19 到序号 20 的转变与以上三种情况原因相同。

表3 总维修成本与可靠度阈值的关系。

\begin{tabular}{llllllllll}
\hline 序号 & 参数 $\boldsymbol{\mu}$ & 参数 $\boldsymbol{\alpha}$ & $\mathrm{t} 1 / \mathrm{h}$ & $\mathrm{t} 2 / \mathrm{h}$ & $\mathrm{t} 3 / \mathrm{h}$ & $\mathrm{t} 4 / \mathrm{h}$ & $\mathrm{t} 5 / \mathrm{h}$ & $\mathrm{t} 6 / \mathrm{h}$ & Zmin/千元 \\
\hline 1 & 0.3 & 0.5 & 1059 & 1371 & 1787 & 2582 & -- & -- & 1.2 \\
2 & 0.3 & 0.6 & 1059 & 1371 & 1787 & 2582 & -- & -- & 1.2 \\
3 & 0.3 & 0.7 & 1059 & 1615 & 1927 & 2528 & -- & -- & 1.2 \\
4 & 0.3 & 0.8 & 312 & 1107 & 1659 & 2528 & -- & -- & 1.2 \\
5 & 0.3 & 0.9 & 715 & 1131 & 1687 & 2343 & 2582 & -- & 1.3 \\
6 & 0.2 & 0.5 & 1059 & 1638 & 2194 & 2582 & -- & -- & 1.2 \\
7 & 0.2 & 0.6 & 1059 & 1638 & 2194 & 2582 & -- & -- & 1.2 \\
8 & 0.2 & 0.7 & 670 & 1375 & 1791 & 2582 & -- & -- & 1.2 \\
9 & 0.2 & 0.8 & 312 & 1107 & 1659 & 2582 & -- & -- & 1.2 \\
10 & 0.2 & 0.9 & 715 & 1222 & 1778 & 2343 & 2582 & -- & 1.3 \\
11 & 0.1 & 0.5 & 1059 & 1778 & 2194 & 2582 & -- & -- & 1.2 \\
12 & 0.1 & 0.6 & 1059 & 1778 & 2194 & 2582 & -- & -- & 1.2 \\
13 & 0.1 & 0.7 & 670 & 1465 & 2017 & 2582 & -- & -- & 1.2 \\
14 & 0.1 & 0.8 & 312 & 1107 & 1659 & 2582 & -- & -- & 1.2 \\
15 & 0.1 & 0.9 & 715 & 1222 & 1778 & 2343 & 2582 & -- & 1.3 \\
\hline
\end{tabular}




\begin{tabular}{llllllllll}
\hline 序号 & 参数 $\mu$ & 参数 $\alpha$ & $\mathrm{t} 1 / \mathrm{h}$ & $\mathrm{t} 2 / \mathrm{h}$ & $\mathrm{t} 3 / \mathrm{h}$ & $\mathrm{t} 4 / \mathrm{h}$ & $\mathrm{t} 5 / \mathrm{h}$ & $\mathrm{t} 6 / \mathrm{h}$ & $\mathrm{Zmin} /$ 千元 \\
\hline 16 & 0.4 & 0.5 & 1059 & 1371 & 1787 & 2582 & -- & -- & 1.2 \\
17 & 0.4 & 0.6 & 1059 & 1371 & 1787 & 2582 & -- & -- & 1.2 \\
18 & 0.4 & 0.7 & 1059 & 1615 & 1927 & 2582 & -- & -- & 1.2 \\
19 & 0.4 & 0.8 & 715 & 954 & 1103 & 1659 & 2582 & -- & 1.3 \\
20 & 0.4 & 0.9 & 507 & 670 & 1086 & 1642 & 2343 & 2582 & 1.4 \\
\hline
\end{tabular}

参数 $\alpha$ 的较准确的临界值需要进行更精确的分析, 本 文展示了当 $\mu=0.3$ 时的分析结果。

从表 4 中可知, 当 $\mu=0.3$ ，参数 $\alpha$ 为区间 [0.84, 0.85] 中的某个值时, 维修成本就会发生变化, 由于参数 $\alpha$ 过大造
成了过度维修。同时, 从表4可以看出, 即使维修成本相同, 维修时刻也可能不同，其可能是有多种解，多种任务排序， 不同的任务排序对维修时刻产生影响, 或者可能是参数 $\alpha$ 对 其任务排序会造成影响, 本文就不对这方面进行分析。

表4 维修阈值的进一步分析。

\begin{tabular}{|c|c|c|c|c|c|c|c|c|}
\hline 序 号 & 参数 $\mu$ & 参数 $\alpha$ & $\mathrm{t} 1 / \mathrm{h}$ & $\mathrm{t} 2 / \mathrm{h}$ & $\mathrm{t} 3 / \mathrm{h}$ & $\mathrm{t} 4 / \mathrm{h}$ & $\mathrm{t} 5 / \mathrm{h}$ & Zmin/千元 \\
\hline 1 & 0.3 & 0.80 & 312 & 1107 & 1659 & 2528 & $\begin{array}{l}-- \\
\end{array}$ & 1.2 \\
\hline 2 & 0.3 & 0.81 & 312 & 1107 & 1659 & 2528 & -- & 1.2 \\
\hline 3 & 0.3 & 0.82 & 312 & 1107 & 1659 & 2528 & -- & 1.2 \\
\hline 4 & 0.3 & 0.83 & 312 & 1107 & 1659 & 2528 & -- & 1.2 \\
\hline 5 & 0.3 & 0.84 & 312 & 1107 & 1659 & 2528 & -- & 1.2 \\
\hline 6 & 0.3 & 0.85 & 507 & 1059 & 1615 & 1927 & 2582 & 1.3 \\
\hline 7 & 0.3 & 0.86 & 507 & 1059 & 1615 & 2017 & 2582 & 1.3 \\
\hline 8 & 0.3 & 0.87 & 715 & 1131 & 1687 & 2343 & 2582 & 1.3 \\
\hline 9 & 0.3 & 0.88 & 715 & 1131 & 1687 & 2343 & 2582 & 1. 3 \\
\hline 10 & 0.3 & 0.89 & 715 & 1131 & 1687 & 2343 & 2582 & 1.3 \\
\hline 11 & 0.3 & 0.90 & 715 & 1131 & 1687 & 2343 & 2582 & 1.3 \\
\hline
\end{tabular}

\section{6. 结论}

本文提出了单设备的基于役龄分析的多种维修方式 的维修调度优化模型, 通过实例可知, 一般情况下, 任务 总量相同时，采用多种维修方式进行设备维修，会降低维 修成本。同时, 随着任务规模和任务总量的增加, 维修成 本也会增加。维修阈值会对维修成本、维修次数以及维修 时间产生影响, 但根据目前实例来看, 在任务规模小的情 况下，影响不太显著。

本文未对大规模任务进行研究, 主要是受到计算方法 的限制, 所以在以后会对算法进行改进。维修阈值的选择 或不同维修方式的单位成本对总体维修成本和维修计划 的影响等问题需要更深入的研究。

\section{致谢}

本文为上海市浦江人才计划 (15PJ1402800) 的阶 段性成果之一。

\section{参考文献}

[1] Ba Kader, Dellagi Sofiene, Rezg Nidhal, and Erray Walid. Joint optimization of preventive maintenance and spare parts inventory for an optimal production plan with consideration of $\mathrm{C0} 2$ emission [J]. Reliability Engineering \& System Safety, 2016, 149: 172-186.
[2] Lei Xiao, Sanling Song, Xiaohui Chen, and David W. Coit. Joint optimization of production scheduling and machine group Preventive maintenance [J]. Reliability Engineering \& System Safety, 2016, 146: 68-78.

[3] 金玉兰, 蒋祖华, 侯文瑞. 以可靠性为中心的多部件设备预 防性维修策略的优化 $[J]$. 上海交通大学学报, 2006。

[4] Morad Assid, Ali Gharbi, Adene Hajji. Joint production, setup and preventive maintenance policies of unreliable two-production manufacturing systems [J]. International Journal of Production Research, 2015, 53 (15): 1-16.

[5] 喻明让, 张英杰, 陈琨, 高瑞, 张定. 考虑调整时间的作业车 间与预防性维修集成方法 [J]. 西安交通大学学 报, 2015, 49 (6) : 16-21。

[6] 崔维伟, 陆志强. 单机系统的生产调度与预防性维护的集成 优化 $[J]$. 上海交通大学学报, 2012, 46 (12) : 2009-2013。

[7] 金玉兰, 蒋祖华. 预防性维修计划和生产调度的多目标优化 [J]. 哈尔滨工程大学学报, 2011, 32 (9) : 1205-1209。

[8］王世进, 生产调度与维护集成的多目标Lorenz非劣遗传优 化 [J]. 工业工程与管理, 2012, 17 (2) : 1-7。

[9］石慧, 曾建潮. 基于寿命预测的预防性维护维修策略 [J]. 计 算机集成制造系统, 2014, 20 (5):1133-1140。

[10］夏唐斌, 奚立峰, 李斯克. 基于RCM的港口机械关键设备的维 修决策 $[J]$.工业工程与管理, 2009, 14 (2) :67-72。 
[11] 张悦民, 杨荔贤. 预防维修周期的一种多目标最优化模型 [J]. 兰州理工大学学报, 2011, 37 (3) : 138-143。

[12] 王远航, 邓超, 胡湘洪, 高军, 黄创绵. 基于多故障模式的复 杂机械设备预防性维修决策 $[\mathrm{J}]$. 计算机集成制造系 统, 2015, 21 (9) : 2504-2514。

[13] 米根锁, 张风霞, 魏蕾. 基于剩余寿命的铁路轨道电路调整 型维修方法研究 [J]. 铁道学报, 2015, 37 (4) :69-74。

[14] 宋之杰, 常建美, 侯贵宾. 基于可靠性的多部件港口设备机 会维修模型 $[J]$. 现代制造工程, 2015 (7) : 130-136。
[15] Malik Mazhar Ali Khan. Reliable preventive maintenance policy [J]. AIIE Transactions. 1979, $11(3)$ : 221-228.

[16] Nakagawa Toshio. Sequential imperfect preventive maintenance policies $[\mathrm{J}]$. IEEE Transactions on Reliability, 1988, 37 (3) : 295-298.

[17] 同济大学数学系. 高等数学 [M]. 北京: 高等教育出版 社, 2014:234-235。 